\title{
ZU MENSCH UND MENSCHENÄHNLICHKEIT BEI WALTER BENJAMIN
}

PHILIPP NOLZ

\begin{abstract}
Benjamin's philosophy has often been discussed without considering the sensible nature of thinking and the peculiar role the individual obtains in this conception from his early writings on. This article wants to point out the key role of the individual between sensibility and perception through language.
\end{abstract}

\section{Einleitung}

Im folgenden Artikel soll herausgearbeitet werden, wie und vor welchem Hintergrund bei Benjamin vom menschlichen Individuum die Rede ist und wie im Weiteren der Mensch seine Ähnlichkeit zu sich selbst als Bestimmung erhält beziehungsweise auch verlieren kann. Dem Anschein dieses weitgehenden Projekts zum Trotz sollen die Ausführungen sowohl das Problem einer ästhetischen Bestimmung des Menschen auf der Grundlage der Wahrnehmung wie auch seiner Erkenntnisbedingungen in der Sprachphilosophie herausstellen, um dabei die beiden Sphären in ihrer gegenseitigen Durchdringung zueinander aufzuzeigen und aufrechtzuerhalten. Gerade in dieser Hinsicht scheint der Begriff des Individuums von zentraler Bedeutung, anhand dessen Analyse zuerst das philosophische Fundament des bürgerlichen Sprachverständnis und die Rolle des Individuums mit Benjamin kritisiert werden soll, ehe im weiteren Verlauf eine Art Restitution des Begriffs des Individuums versucht wird.

Diese argumentative Bewegung ist zunächst der eigentümlichen Verfasstheit des Individuums geschuldet, welches als empirisch-psychologisches Wesen der 
natürlichen Zufälligkeit der Umstände, in denen es sich findet, unterworfen ist und dessen Singularität sich der Erkenntnis immer zu entziehen droht. Andererseits ist die Wahrung einer uneinholbaren Einzigartigkeit des Daseins, wie sie im Begriff des Individuums mitschwingt, aufgrund ethischer Belange die Bedingung dafür, dass sich die Form des Denkens nicht gegen das einzelne Leben richtet. Die Verantwortung für das eigene Handeln und Sprechen etwa, wie auch die Rechtfertigung des Schutzes der Integrität und Unversehrtheit des Einzelnen entsprächen solchen ethischen Überlegungen. So wie ein System des Denkens, wo es einem Inventar sich angleicht, den vielfältigen Ausdrücken des Lebens keinen Platz gibt, so ist das Einzelne, wo es als bloß vereinzeltes Leben gelebt wird, jeder übersinnlichen Bestimmung enthoben und verfällt infolgedessen der Bedeutungslosigkeit. Als zweifacher Grenzbegriff soll also das Individuum verstanden werden, wenn sein Begriff verteidigt werden soll.

Vor dem Hintergrund der frühen Auseinandersetzung Benjamins mit der Philosophie Kants und dem Neukantianer Hermann Cohen sollen zuerst die sprachphilosophischen Grundlagen umrissen werden, die es in weiterer Folge ermöglichen, das bürgerliche Sprachverständnis hinsichtlich des Begriffs des Individuum $\mathrm{zu}$ kritisieren und somit die fragwürdige Annahme eines außerhalb der Sprache und der - wie sich noch zeigen wird - Erkenntnis stehenden Begriffs des Menschen zurückzuweisen.

Ausgehend von einer solchen Auflösung des bürgerlichen Begriffs des Individuums in der Sprache wird sodann eine Konkretisierung des Menschen qua einzelnes Dasein aus der Sprache heraus versucht. Dieses Vorgehen hat einen doppelten Grund, der vorerst in der Gegenüberstellung zweier Ansprüche formuliert werden kann: der Mensch muss als Einzelner aus einem umfassenden Zusammenhang hervorgehen, denn ein solcher ermöglicht die Bindung an ein Gemeinsames, wodurch sowohl erkenntnistheoretische Verbindlichkeit wie auch die Möglichkeit ethischen Handelns im oben erwähnten Sinn bedingt werden. Andererseits muss das Einzelne sich gegen eine bloße Allgemeinheit positiver Bestimmungen, also gegen die gegebene Sprache behaupten, um als Individuum Einzigartigkeit und Gemeinschaft zu verbinden, eine - wie Benjamin schreibt - „unteilbare aber unabgeschlossene Einheit" zu bilden. ${ }^{1}$

Dieser Sachverhalt wird sich vor allem aus den Aufzeichnungen zum „Problem der Wahrnehmung" skizzieren lassen, da die dort getroffene Unterscheidung zwischen Leib und Körper sowie ihrer gegenseitigen Bezugnahme im Begriff des

1 Benjamin, Walter: Fragmente, Autobiographische Schriften, Suhrkamp, Gesammelte Schriften Bd. VI, Frankfurt/Main 1991 S. 71 (im Weiteren zitiert als GS+Band+Teilband). 
Daseins einen zentralen Lektüreschlüssel an die Hand gibt, mit der das Motiv der Bestimmung des Menschen unter historisch-ästhetischen (im weiten Sinne der Wahrnehmung; alı $\theta \eta \sigma ı \varsigma) ~ G e s i c h t s p u n k t e n$ für eine weitergehende Auseinandersetzung mit den späteren Schriften Benjamins vorbereitet werden soll.

\section{Das Problem des Menschen in der Sprache}

Bereits in den frühen Texten ab 1916, in denen Benjamin seine Sprachphilosophie zu formulieren suchte, konstatiert er, dass die „bürgerliche Auffassung der Sprache "2 unredlich sei, da sich darin zwei festgestellte oder fixierte Positionen starr gegenüber stünden, um untereinander bloß Erkenntnisdaten auszutauschen, die Position des Sprechenden also nicht auf deren Bedingtheit durch die Sprache selbst rückbezogen wird.

Zuvorderst verweist Benjamin dabei auf den mangelhaften Begriff eines Gebrauchs der Sprache („... es gibt also keinen Sprecher der Sprache, [...] der durch diese Sprache sich mitteilt." $)^{3}$, um zu zeigen, dass eine solche Sprachphilosophie auf grundsätzlichen Verkennungen sprachlichen Ausdrucks sowie einer kaum einsichtig zu machenden epistemologische Grundlagen beruht. Benjamin hält dagegen fest, dass die Sprache nicht bloß ein etwas zwischen zwei außerhalb sprachlicher Beziehungen stehender Subjekte sei, der Mensch also unmöglich „eine Sache anderen Menschen mit[teilt] " ${ }^{\text {, }}$ ohne sich selbst mitzuteilen. Der Mensch kann sich dieser These folgend nicht äußern, ohne sich selbst auszudrücken, ohne sich selbst eine Bestimmung als Aussagender zu geben. Anders gewendet bedeutet dies, dass der Mensch als einzelner Sprechender nur durch sein Sprechen bestimmbar ist, das in der/einer Sprache geschieht Das bürgerliche Sprachverständnis ist deshalb unredlich, weil es nicht einzuholen vermag, was es unablässig vorgeben muss, zu tun: Wer spricht, der greift nicht bloß auf ein gegebenes Instrument zurück, das er beliebig anwenden könnte und dabei größeren und kleineren Erfolg hätte, sondern fixiert die Umstände oder die Dinge um ihn herum, sodass diese zu seinen Bestimmungen werden. Eine Notiz zu diesem Sprachdenken erhellt hier die Fragestellung Benjamins, wenn dieser sich fragt, wie sprachlich verfasstes Denken aufzufassen sei. Er schreibt dazu: „Aber kann ein Mensch als empirisches Wesen überhaupt denken? Ist Denken überhaupt in dem Sinne eine Tätigkeit wie

\footnotetext{
GSII-1, S. 144.

Ibid., S.142.

Ibid., S. 144.
} 
hämmern, nähen, oder ist es keine Tätigkeit auf etwas hin, sondern ein transzendentes Intransitivum, wie gehen ein empirisches?"

Das Individuum hat hier für Benjamin - so scheint es - hinsichtlich seiner sprachlich fundierten Erkenntnistheorie keinen anderen Geltungsanspruch als jenen, ein Subjekt der Aussage zu sein. Alle anderen Bestimmungen bleiben konsequenterweise an dieser Stelle noch außen vor. Wenn demnach davon die Rede war, dass die Sprache Medium sei, dann lässt sich darunter dieses „transzendente Transitivum“ verstehen, worin prädikative Aussagen erst durch ihre nachträgliche Gruppierung den Platz für die sprachliche (Re)Konstruktion des Menschen erlauben, der sie ausgesprochen hat. ${ }^{6}$ Demgegenüber wird die bürgerliche Auffassung gerade deshalb kritisiert, weil das Individuum dort - als Komplement des Begriffs der Sprache als Kommunikationsmittel d.h. Mitteilung - einem naturalisierten Begriff entspricht, dessen Bestimmungen dem sprachlichen Ausdruck vorausgehen müsste, und gleichzeitig durch die Sprache vollständig erkennbar wäre.

Etwas verkürzt gesagt, gibt es für Benjamin in der kritisierten Sprachauffassung ein konstitutives Zweiergespann zwischen Kommunikationsmittel (Mitteilung) und Rezipient (Individuum), wodurch die erkenntnistheoretischen Grundlagen auf einem naturalistischen Menschenbegriff und einer konventionalistischen Sprachphilosophie beruhen. Die Natur wird darin außersprachlich fixiert, die Sprache zu einem Oberflächeneffekt, dessen Funktion in übereingekommenen Gebrauch der Bezeichnungen liegt.

Als Gegenbegriff zum diesem Sprachverständnis könnte folglich, wie Benjamin andeutet, eine "mystische Sprachtheorie "7 formuliert werden, die den einzelnen Menschen in ein bloßes Epiphänomen verwandelt und in einem wie auch immer gearteten Begriff der Totalität des Lebens oder der Natur auflösen möchte. ${ }^{8}$ Es wäre demnach der Mensch ein bloß Gesprochenes, ein einfacher Ausdruck der Sprache, die ihrerseits nun den Status des Pseudo-Subjekts erhielte. Eine solche mythische Sprachtheorie scheint jedoch in all ihrer Radikalität ebenso unhaltbar, da das in der Sprache selbst Einzigartige am Menschen, sich selbst als Subjekt der

GSVI, S. 43.

6 Vgl. Menninghaus, Winfried: Walter Benjamins Theorie der Sprachmagie, Suhrkamp, Frankfurt/ Main 1995, S. 85f.

GSII-1, S. 150.

8 Als theoretische Referenz gilt hier Benjamins Kritik an der Lebensphilosophie, insbesondere jener Ludwig Klages. Siehe hierzu: Weigel, Sigrid: „Treue, Liebe, Eros. Benjamins Lebenswissenschaft“, in Deutsche Vierteljahrsschrift für Literaturwissenschaft und Geistesgeschichte, Ausgabe 4, J.B. Metzler, Stuttgart 2010, S. 580-596; siehe auch Jesi, Furio: Mito, Arnoldo Mondadori Editore, Milano 1980, S. $105 f$. 
Aussage zu setzen (in der Form der „benennende[n] Sprache“9), über alle Maße verallgemeinert und damit einer spiritualisierten und differenzlosen Natur zugeschlagen würde.

Wenngleich auch Benjamins Sprachphilosophie durchaus Anleihen an unterschiedlichen Formen der Mystik nimmt ${ }^{10}$, so insistiert er dennoch auf die grundsätzliche Scheidung zwischen Sprache als Erkenntniszusammenhang und Sprache als bildlicher Ausdruck der Natur bzw. des Lebens. ${ }^{11}$ Diese Unterscheidung ermöglicht es Benjamin, dem Menschen einerseits erkenntnistheoretisch zu qualifizieren, nämlich durch die Funktion der benennenden Sprache, und andererseits die produktive und offene Erfahrung lebendiger Auseinandersetzung mit dem in der Sprache Gegebenen zu beschreiben - die Erfahrungen, die sich durch die Benennung der Dinge und ihrer reflexiven Determination des Menschen ergeben, ist exakt die Erfahrung der Medialität der Sprache selbst: Da der Mensch im Sprechen handelt, ist die prädikative Selbstzuschreibung - die Antwort auf die Frage, was der konkrete Mensch sei - über die ihn umgebenden Dinge vermittelt; der Mensch als Erscheinung ist für Benjamin immer sekundärer oder retroaktiver Effekt seiner eigenen Tätigkeit des Benennens. ${ }^{12}$ Auf der anderen Seite kann diese Tätigkeit des Benennens nie im luftleeren Raum geschehen, sondern geht immer von einem bestehenden Begriff des Menschen aus, der im konkreten Falle benennt, oder wie Benjamin in seiner Beschreibung des Individuums schreibt: „Das historische Leben vergeht immer an irgendeinem Ort". ${ }^{13}$

Das Benennen als erzeugende Tätigkeit für die Erkenntnis und Konfiguration einer konkreten „Materialität"14 in der Erkenntnis stellt die dialektische Bewegung des Sprachdenkens dar. Wurde ersteres bis hierher als die sprachliche Grundlegung des Erkenntnisprozesses beschrieben, wird nun diejenige Ebene behandelt, die sowohl als Ausgangspunkt (,irgendein bestimmter Ort“) diesem Erkenntnisprozess zugrunde liegt wie auch dessen Erzeugnis bildet. Es handelt sich dabei um „eine der höchsten Sprachschichten“15, nämlich jene der Wahrnehmung.

\footnotetext{
GSII-1, S. 143.

Menninghaus: Walter Benjamins Theorie, op.cit., S. $195 \mathrm{ff}$.

1 GSVI, S. 34; siehe auch: Deuber-Mankowsky, Astrid: Der frühe Walter Benjamin und Hermann Cohen. Jüdische Werte, Kritische Philosophie, Vergängliche Erfahrung, Vorwerk, Berlin 2000, S. $88 \mathrm{f}$

12 Vgl. Hein, Matthias: Walter Benjamins Konzept der Lektüre, Könighausen \& Neumann, Würzburg 2011, S. $22 \mathrm{f}$.

13 GSVI, S. 71.

14 Deuber-Mankowsky: Der frühe Walter Benjamin, op.cit., S. 92.

15 GSVI, S. 67.
} 


\section{Die Sprachschicht der Wahrnehmung}

Da Benjamin in Nähe und Abgrenzung zu Kant dessen Erkenntnislehre auf sprachlichen Grundlagen neu ausrichtete, sind auch tiefgreifende Umbildungen in Bezug auf die Frage nach der Sinnlichkeit verbunden. Das Individuum als außersprachliche Setzung eines widersprüchlichen Sprachverständnisses wurde in diesem Prozess gewissermaßen „entsinnlicht“, wenn darunter verstanden wird, dass die Natur oder Sinnlichkeit keine vom (Sprach)Denken unabhängigen Erkenntnisstamm bildet, wie es noch bei Kant der Fall war. „Es ist nämlich gar nicht zu bezweifeln“, so schreibt Benjamin, „daß in dem Kantischen Erkenntnisbegriff die wenn auch sublimierte Vorstellung eines individuellen leibgeistigen Ich welches mittels der Sinne die Empfindungen empfängt und auf deren Grundlage sich seine Vorstellungen bildet die größte Rolle spielt. Diese Vorstellung ist jedoch Mythologie und was ihren Wahrheitsgehalt angeht jeder andern Erkenntnismythologie gleichwertig". ${ }^{16}$

Die Vorstellung, von der Benjamin spricht, betrifft die Problematik, dass sich wiederum - wie in der bürgerlichen Sprachauffassung - ein empirisch konstituiertes Ich in starrer Opposition zu den Gegenständen, die dessen Sinne affizieren, befindet. Wenn die Form der Gegenstände aber, aufgrund der kantischen Apriorität der Anschauungsformen, zeitlos festgelegt ist, so ist es auch das menschliche Individuum. In einer kleineren Schrift Kants wird dies ausdrücklich diskutiert, wobei es darin vor allem um die Notwendigkeit der Kontinuität der Natur zum Zwecke der zeitlosen Gültigkeit der Erkenntnis über die menschliche Natur geht. Kant behauptet, „daß, wenn [...] der Künstelei des Menschen [...] ein Vermögen zugestanden würde, [...] das uranfängliche Modell der Natur umzuformen, oder durch Zusätze zu verunstalten, die gleichwohl nachher beharrlich in den folgenden Zeugungen aufbehalten würden, man gar nicht mehr wissen würde, von welchem Originale die Natur ausgegangen sei [...]“. ${ }^{17}$ Es liegt in dieser Argumentation auch ein zentraler Grund, aus welchen erkenntnistheoretischen Gründen sich Kant bemüßigt gefühlt hat, den Begriff der Menschenrasse wissenschaftlich zu verteidigen, nämlich zum Zwecke der Festlegung eines empirisch belastbaren Begriffs des Individuums. Und es ist gerade geschichtlich die Erfahrung Benjamins, auch in expliziter Bezugnahme auf sein Verhältnis zum Judentum, sich einem solchen naturalisierenden Mythos des Menschen philosophisch entgegenzustellen. ${ }^{18}$

16 GSII-1, S. 161.

17 Kant, Immanuel: Gesammelte Schriften, Walter de Gruyter, Akademieausgabe Werke Bd. VIII, Berlin 1928, S. 97.

18 Siehe: Deuber-Mankowsky: Der frühe Walter Benjamin, op. cit., S. 293. 
Vor diesem Hintergrund sieht Benjamin nun auch die Wichtigkeit, die Wahrnehmung in einer Weise zu konzipieren, in der sie als Medium nicht von der Sprache losgelöst wird, sondern in der Sprache als eine Reflexionsstufe erfassbar wird und somit Denken und Anschauung aus ein- und demselben Erkenntnisstamm, nämlich der Sprache, erzeugt werden können. Diese Operation lässt folglich keine strikte Trennung von Denken und Anschauung mehr zu, weil die Anschauung durch das Denken selbst hervorgebracht wird und keine Referenz auf ein wie auch immer geartetes, natürlich „Gegebenes“ mehr möglich ist.

Es wurde jedoch zuvor erwähnt, dass die Sprache und damit auch die Konstitution des Individuums keine reine, transzendentale Bestimmung hat, sondern sich von einem in der Sprache Gegebenen ausgehend transformieren muss. Anders gesagt ist jede Sprache die Gesamtheit der Bedingungen wirklicher Erfahrung, die unhintergehbar dem Sprechen zugrunde liegen, die jedoch im Akt der Aktualisierung niemals bloß mechanisch reproduziert werden, sondern aufgrund ihrer bildlichen Rekonfiguration im Sprechen anderen und durchaus neuartigen Ausdruckszusammenhängen Raum geben und somit jene materiellen Bedingungen verändern, die das Sprechen voraussetzt.

Die Wahrnehmung ist dabei für Benjamin die Sprachschicht, die am deutlichsten über die Weisen aufklärt, in welcher Form Wahrnehmungsinhalte in der Sprache gegeben sind. Die Wahrnehmung besitzt damit eine zentrale geschichtliche Funktion, weil in den verschiedenen Zugängen zur Wahrnehmungswelt ein - wie Benjamin später schreibt - „Archiv unsinnlicher Ähnlichkeiten“"19 konstituiert wird, welches in der Sprache aufbewahrt wird und welches über das Singuläre einer geschichtlichen Epoche aufzuklären vermag, selbst wenn deren Welt fast vollständig verschwunden ist. Die Wahrnehmung, philosophisch durchdrungen,

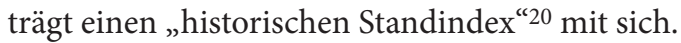

Diese sprachliche Bildlichkeit, von der in der Wahrnehmung die Rede ist, bedarf jedoch eines feinfühligen Lesers, da eine Rekonstruktion, die glaubt, nur auf das Faktische abzustellen, ohne dem Ausdruck selbst Aufmerksamkeit zu schenken, zum Agenten mythischer Gewalt wird. Die eigene bzw. gegenwärtige Wahrnehmungswelt, wo sie sich auf Vergangenes bezieht, muss laut Benjamin die Intention auf das Gemeinte durch die "Art des Meines“21 in sich reflektieren. Denn wenn die Eigenheit einer vergangenen Epoche durch den heutigen

19 GSII-1, S. 208.

20 GSII-2, S. 455. Diese Formulierung taucht später immer wieder auf, beispielsweise in Bezug auf Baudelaire, in einem Brief an Horkheimer bezüglich Gottfried Keller oder, in leicht abgeänderter Form, in der zweiten geschichtsphilosophischen These.

21

GSIV, S. 14 . 
Sprachgebrauch beschrieben wird, wird gegen die Einzigartigkeit des Vergänglichen (einer Epoche) gewaltsam vorgegangen, indem die Geschichte in die sprachlich verfasste „bleibende Erkenntnis“ gepresst, die Erfahrung des radikal Endlichen, wie etwa das Gefühl für das Schöne innerhalb einer Epoche ${ }^{22}$, einer allgemeinen Erkenntnis über die Epoche geopfert wird. Weil aber jeder Sprecher einer Sprache in einem Verweiszusammenhang erst hervortritt, muss einsichtig gemacht werden, von wo ausgegangen wird und wie eine reflexive Bezugnahme auf die eigenen Grundlagen möglich ist. Hierfür bringt Benjamin eine Dialektik zwischen Leib und Körper ins Spiel.

\section{Leib und Mythos}

Benjamin bezeichnet hinsichtlich der Wahrnehmung den Leib als jene Sphäre der Wahrnehmung, die "allem Realen aus seiner Gegenwartsbezogenheit zum geschichtlichen Prozess" 23 zukommt. Diese Bezogenheit bezeichnet die Wahrnehmung als Feld der Erscheinungen, auf die sich der Mensch sprachlich bezieht. Da ein Sich-Beziehen miteinschließt, dass dasjenige, worauf sich bezogen wird, (chrono)logisch vorausgesetzt und als differentielle Beziehung affirmiert wird, wird der Leib gesetzt, begrenzt und als „Gestalt des Geschichtlichen“24 vereinzelt. Der Leib ist also - als eine Konfiguration der virtuellen Gesamtheit von Erscheinungen unter einer geschichtlichen Idee - „Individualität“ und steht damit dem Prinzip nach „höher als die einzelner leiblicher Individualitäten“. ${ }^{25}$ Als Vereinzeltes im Geschichtsprozess heißt das, dass der Leib nicht mit dem Ablauf des Geschichtsprozesses selbst zusammenfällt, sondern dessen immanente Voraussetzung ist - als „Gestalt des Geschichtlichen“ ist der Leib nicht der Geschichtsprozess, sondern das „jeweilige In-ihm-stehen“. ${ }^{26}$

Die Feststellung, dass die Gesamtheit von begrenzten Extensionen d.h. Phänomenen nicht mit dem geschichtlichen Prozess als solchem zusammenfällt, ist von zentraler Bedeutung, da - negativ formuliert - die Gleichsetzung der gegenwärtigen Beziehung auf Vergangenes mit der Geschichte selbst zur Folge hätte, dass das Gegebene unabhängig einer sprachlichen Bezugnahme gedacht würde

\footnotetext{
22 Siehe zu der Debatte über eine immanente Auslegung der Kunst einer Epoche bei Benjamin: Caygill, Howard: „Walter Benjamin's concept of cultural history“, in Ferris, D. (Hrsg.), The Cambridge Companion to Walter Benjamin, University Press, Cambridge 2004, S. 73-96.

23 GSVI, S. 79.

24 Ibid., S. 78.

25 Ibid., S. 80.

26 Ibid., S. 78.
} 
und somit von geschichtlichen Veränderungen losgelöst erschiene. Diese Gefahr als Ausgangspunkt anerkennend schreibt Benjamin, es gäbe „eine Geschichte der Wahrnehmung, welche zuletzt die Geschichte des Mythos ist". 27

Benjamins Vorgehen zeichnet sich aber nicht in einer bloßen Zurückweisung des Mythischen aus, wie seine immer wieder geäußerte Kritik an einer naiv sich gebärenden Aufklärung im Umgang mit religiösen oder kultischen Elementen gesellschaftlichen Lebens zeigt. Es wird vielmehr versucht, den mythischen Gehalt und der reflexiven Haltung, welche den Mythos sowohl erzeugt wie sich auch als Bestimmung und Abgrenzung auf ihn bezieht, in der Bewegung des Denkens selbst einzufangen. Der Mythos ist demzufolge für das Denken immer zurückgelassene Vergangenheit, der aufgrund der genannten Dynamik nie als Aktualität erscheint, wohl aber in der operativen Funktion bestehen bleibt. Die Geschichte der Wahrnehmung kommt „aus Veränderungen des Leibes zustande, aber erst sie [die Geschichte; Anm. P.N.] gibt diesen die geistige Bedeutung und Krönung [...] im Mythos“.28

Weil das Denken geschichtliche Bewegung ist, krönt sie, wie Benjamin es formuliert, das eben Zurückgelassene im Mythos. Der Mythos ist somit, wo er als Mythos erkannt und in die Bewegung des Denkens als dessen Erzeugnis aufgenommen wurde, nicht so sehr ein fremdes Element, das das Leben durch dessen Fixierung gefährdet, als vielmehr die erste Synthese des Leibes oder der Erscheinungswelt ${ }^{29}$, die als Ausgangspunkt und in der reflexiven Bezugnahme das Denkens selbst fordert.

Problematisch wird der Mythos genau dort, wo das Gegebene für das Denken - in Bezug auf die Sprache sind das die verschiedensten Sprachbilder als Elemente dieses Denkens - zu einem Allgemeinen und nicht durch das Denken selbst Erzeugtes wird. Dieser Zusammenhang, der sich oben in den sprachphilosophischen Grundlagen ausweisen ließ, wird nun vor dem Hintergrund einer Theorie der Wahrnehmung verwendet, um die Beziehung zwischen der Gegenwartsbezogenheit der Sprache in ihren Wahrnehmungsinhalten (Leib) und der Gegenwart des Denkens selbst herauszuarbeiten. Der Mythos wird nicht dann zu einem, dem Leben feindselig gesinnten Konstrukt, wo er zurückgelassen wird, da diese Fluchtbewegung des Denkens aus dem Gegebenen die mythischen Elemente selbst

\footnotetext{
Ibid., S. 67.

Ibid.

29 Benjamin konzipiert den Mythos explizit als ersten Ausweg des Menschen aus einer prähistorischen „Sumpfwelt", eine Ausweg jedoch, der seinerseits von seiner gewaltvollen Beherrschung dieser Vorwelt befreit werden muss. Vgl. Lebovic, Nitzan, „Benjamins ,Sumpflogik“, in Weidner, Daniel, Profanes Leben. Walter Benjamins Dialektik der Säkularisierung, Suhrkamp, Frankfurt/Main 2010, S. 207-212. Siehe auch: Jesi, F., Mito, op.cit., S. 107f.
} 
durch die „Modification von Gestalt zu Gestalt“30 produktiv in neue Konstellationen bringen kann, die den Mythos sowohl als integralen Bestandteil enthalten, wie sie dadurch auch dessen Gewalt zu bannen vermögen. Stéphane Mosès wies bereits die Zentralität dieser Denkfigur im Werk Benjamins auf, wonach die imaginative Modifikation des Ausgangspunktes zur selben Zeit eine Deformation der Vergangenheit bedeutet. ${ }^{31}$ Es ist dabei entscheidend hervorzuheben, dass diese Deformation keine Gewalt gegen das Vergangene, sondern Mittel gegen den Mythos ist, der als Immer-Gleiches des Vergangenen die eigentlich deformierte Geschichte darstellt, in seiner Orts- und Zeitlosigkeit die genuine Gewalt gegen ein subjektive Leben bildet. Regressiv geht der Mythos dort vor, wo er mit dem erwähnten geschichtlichen Prozess selbst zusammenfällt, wo also Gegenwartsbezogenheit und Gegenwart des Denkens in ein zweideutiges Verhältnis, in eine Beziehung der Ununterscheidbarkeit zusammenlaufen.

Dies ist der Fall, wenn von dem Menschen die Rede ist: als empirisches d.h. phänomenales Wesen ist vom Menschen bloß bekannt, was ihm nicht mehr gegenwärtig ist. Eine mythische Bestimmung des Menschen, welche mit jeder historisch-anthropologischen Untersuchung einhergeht, unterscheidet sich vom „lebendigen" Menschen also nicht dadurch, dass letzterer nicht in der Welt sei, nicht erscheint, da der Mensch nur als Erscheinung in der Wahrnehmung zu denken ist. Wie oben bereits gezeigt, ist es die jeweilige Bezugnahme, die Art des Meinens, welche ausschlaggebend dafür ist, ob der Mythos in die geschichtliche Bewegung aufgenommen wird und somit in seiner produktiven Veränderung gedacht werden kann - wodurch er zum Leib wird - oder ob geronnene Bestimmungen über den Menschen gewaltsam gegen die sich ändernden Umstände des gesellschaftlichen Lebens durchgesetzt werden, ob also der Mythos (als Aufgegebenes) Mythos (als historische Totalität) bleibt.

Diese Logik zeigt sich in Benjamins Texten auch später noch, etwa wenn er im Aufsatz „Erfahrung und Armut“ vom Ende der Menschenähnlichkeit spricht. Um auf eine solche Lektüre hin jedoch noch Klarheit zu schaffen, bleibt noch eine Klärung erforderlich, die zur Bestimmung der Singularität oder der Einzigartigkeit des individuellen Menschen unverzichtbar ist, will man keinem (mythischen) Antihumanismus das Wort reden. Es ist schließlich bis hierher nicht einsichtig geworden, durch welche Instanz sich das Denken vom unmittelbar Gegebenen lösen kann, um dieses im Leib als Mythos bannen zu können.

30 GSVI, S. 78.

31 Mosès, Stéphane: Walter Benjamin et l'esprit de la modernité, Les éditions du cerf, Paris 2005. 


\section{Körper gegen den Mythos}

Die geschichtliche Bewegung, von der bereits gesprochen wurde, ist unter einem doppelten Aspekt zu betrachten: zum einen muss dieser Prozess über das bloß Gegebene hinaustreiben, muss die Möglichkeit schaffen, das mythische Ausgangsmaterial zurückzulassen, dabei gleichzeitig auch dieses Material als zurückgelassenen Mythos gewissermaßen hervorbringen. Zum anderen ist es, gerade hinsichtlich der Diskussion über den Menschen, entscheidend zu sehen, wie das Menschliche jenseits des empirisch Lebendigen in diesem Geschichtsprozess eingeht, wie also, um eine Formulierung Hannah Arendts aufzugreifen, die „lebendige Menschlichkeit des Menschen “32 historische Bewegung werden kann oder in einer solchen sich ausdrückt. Zur Klärung dieses Zusammenhangs führt Benjamin den Begriff des Körpers ein.

Der Körper hat dabei einen paradoxen Status inne: er ist „Substanz im Unterschied zum Leib, welcher nur Funktion ist" ${ }^{33}$ Während der Leib eine geschichtliche und endliche Gestalt war, innerhalb derer eine relationale Struktur die Erscheinungen aneinander bindet und begrenzt, ist der „Körper und der ihm zugehörende Geist nicht auf Beziehung sondern auf Dasein schlechthin gegründet" .34 Diese Bemerkung ist insofern schwierig zu verstehen, da der Körper sowohl als ein Absolutes beschrieben wird, das vom Geschichtsprozess nicht betroffen ist, gleichzeitig aber eben die Existenz dieses Geschichtsprozesses selbst versichert, weil dieser auf dem Dasein gründet, das immer geschichtlich sich ausdrückt. Die Schwierigkeit ist somit jene - in aller dialektischen Schärfe formuliert - dass die jeweilige historische Zeitlichkeit durch ein Zeitloses, im Grunde aber Ewiges bedingt wird, dieses Ewige jedoch nicht außerhalb der Zeitlichkeit einfach affirmiert werden kann, weil der Ausgangspunkt der Befragung die endliche Welt der Erscheinungen ist, das Ewige folglich wiederum durch die gegenwärtige Zeitlichkeit hindurch erst ausgewiesen werden muss. ${ }^{35}$

Auf der Grundlage erkenntnistheoretischer Erwägungen wurde oben bereits die Unterscheidung zwischen Erkenntniszusammenhang bzw. Erkenntnis der Erfahrung auf der einen Seite, dem eigentlichen Begriff der Erfahrung (bildlicher

32 Arendt, Hannah: Von der Menschlichkeit in finsteren Zeiten. Rede über Lessing, Piper Verlag, München 1960, S. 17.

33 GSVI, S. 79.

34 Ibid.

35 Die Debatte des Körpers soll sich an dieser Stelle auf die für uns relevanten Punkte beschränken. Es sei hier aber darauf hingewiesen, dass sich bereits zahlreiche Bestimmungen dessen finden, was Benjamin im Begriff des „Urphänomens“ im weiteren Verlauf seiner Tätigkeit einzufangen versucht. Siehe dazu Mosès: Walter Benjamin, op.cit., S. 129ff und S. $146 f$. 
Ausdruck der Sprache) auf der anderen Seite getroffen. Diese Unterscheidung wird nun von Benjamin erneut auf Basis der Wahrnehmung durchexerziert, indem der Erkenntniszusammenhang geschichtlicher Erfahrung durch den Körper als Instanz, die einem relationalen Wissen äußerlich ist, beschrieben wird, denn „[s] oweit wir von Wahrnehmung wissen, wissen wir von unserem Körper [...]“.36 Es handelt sich aber hierbei um ein vermitteltes Wissen der Wahrnehmung durch ein unmittelbares Wissen der Körperlichkeit, das „als Lust und Schmerz“ sich manifestiert und somit „ohne bestimmte gestaltete Begrenzung sich erstreckt“. ${ }^{37}$ Benjamin versucht hier erneut zu umschreiben, wie ein durch sprachliche Beziehung vermitteltes Wissen (wie jenes der Wahrnehmung) im Umkehrschluss auf einer Erfahrung dieses Wissens beruht, welches sowohl in der Sprache sich ausdrückt, wie auch diesen Ausdruck durch die konkrete, mittels einer spezifischen historischen Erfahrung geprägten Bezugnahme auf die Wahrnehmungsinhalte (Leib) konstruiert.

Der Körper kann somit als die erste Negation des Mythos verstanden werden. Setzt letzterer sich als die formelle Gesamtheit des Wissens, negiert der Körper dessen selbstreferentielle Logik und formt ihn zum (objektiven) Leib um. Der Körper als Unmittelbarkeit der Erfahrung erscheint hier nicht als begrenzte Gestalt, ist nicht Teil des Leibes, sondern tritt zuerst als Fremdkörper, Fehler oder Friktion im Ablauf der Wahrnehmungswelt. Die formale Wiederholung eines vollständig determinierten Ganzen, welches in der je aktuellen Wiederholung dem Ganzen nichts Neues hinzufügt als Charakteristikum des Mythos, wird durch den Körper unterbrochen, indem nicht die Wiederholung negiert, sondern die Einzigartigkeit jeder Wiederholung im Dasein, also in der aktualen Wirklichkeit gelebter Erfahrung aus dem indifferenten Strom der Zeitlichkeit gerissen wird und abseits des Inhalts die konkret gegebene Form des Inhalts zum Vorschein gebracht wird. ${ }^{38}$ Doch die Flucht aus dem Mythos ist auf dieser Ebene instabil und bleibt dialektisch an seinen Ausgangspunkt gebunden. Das heißt, dass auf dieser Stufe der subjektive Akt des Versagens, des Verfehlens oder des unvollständigen Reproduzierens gegebener Strukturen in der Wahrnehmung oder im Sprechen noch als residuale Abweichung eines statischen Wissens verstanden werden kann, wie beim bürgerlichen Sprachverständnis erwähnt wurde. Die das Gegebene überschreitende Kraft des Daseins im Begriff des Körpers wäre somit bloß als Misslingen oder Fehler in der ordentlichen Transmission des Wissens gewertet. Benjamin versucht deshalb,

\footnotetext{
6 GSVI, S. 79 (Hervorhebung, P.N.)

Ibid.

38 Siehe zum Verhältnis von Ewiger Wiederkehr und radikal Neuem: Mosès: Walter Benjamin, op. cit., S. 212 .
} 
die wirkliche Möglichkeit der Veränderung in den Ausgangsbedingungen selbst zu verorten. Es ist schließlich ungenügend, den Mythos als Mythos auszuweisen, da er sich selbst nicht versteckt. Das, was durch den Begriff des Körpers bewirkt werden soll, ist eine Widerständigkeit des konkreten Lebens gegenüber jenen Kräften geschichtlicher Überlieferung, die im Mythos gewaltsam wirken. Zu diesem Zweck schreibt er, dass „der Irrtum nicht länger aus dem Irren erklärt werden“ dürfe. ${ }^{39}$

Eine solche Reflexion auf die sprachlichen Bedingungen des Wissens bedarf in weiterer Folge eines Anhaltspunktes in der Sprache, aufgrund dessen die Möglichkeit der Modifikation der Ausgangsbedingungen nachweisbar und neuer Erfahrungen im Alten oder Zurückgelassenen zugänglich wird. Benjamin versucht also zu zeigen, wie der Körper vom Status einer bloßen Störung des mythischen Ablaufs der Wahrnehmung zu einem Vermögen in der Wahrnehmung selbst werden kann, wie also nicht nur inhaltlich, sondern der Form nach eine Mystifizierung des Vergangenen überkommen werden kann. Hier kommt erneut die besondere Sprache des Menschen ins Spiel.

Der Mensch ist in der leiblichen Welt bisher bloße Erscheinung, objektiv gegebene Individualität unter anderen gewesen, wohingegen der Körper als etwas Unmenschliches oder Dämonisches bezeichnet werden kann ${ }^{40}$, als Abweichung von der Norm dessen, was der Mensch - nach den Kriterien des Gewesenen sein solle. Dieses Unmenschliche deutet Benjamin nun dahingehend um, dass er der Individualität des vereinzelten Menschen die „Singularität“ oder „Einzigkeit“ gegenüberstellt, die den Menschen gewissermaßen abseits seines phänomenalen Wesens auszeichnet und dem Körper zugehörig ist ${ }^{41}$ : Der Leib als Matrix einer gegebenen Natur; der Körper als die geistige Natur oder das geistige Wesen, welche der Sprache nicht im Benennen zugänglich ist, sondern erst die Distanz zur „natürlichen Welt" einführt, die eine solche Tätigkeit ermöglicht.

Als Vermögen des Körpers ist diese Einzigartigkeit laut Benjamin die bildliche Hervorbringung lebendiger Erfahrung in Erkenntnis, welche sich durch Konfigurationen im Ausdruck und der damit einhergehenden „mimetischen“ Tätigkeit ursprünglich in der Sprache finden lässt. Eine solche mimetische Kraft ist, wie Menninghaus es formulierte, „in der Sprache aktuell und universell wirksam“, da sie als prägendes Prinzip den Sinnzusammenhang durchwaltet. ${ }^{42}$ Es soll hier aber weniger das konkrete Ausdrucksvermögen untersucht werden, als vielmehr

\footnotetext{
GSII-1, S. 167.

40 Diese Bezeichnungen tauchen systematisch in den Skizzen zur Anthropologie auf (GSVI, S. 64), ziehen sich jedoch über die Abhandlungen zu Goethe und Kraus durch das Werk Benjamins.

GSVI, S. 80f.

Menninghaus: Walter Benjamins Theorie, op.cit., S. 70.
} 
die Frage bezüglich einer - wenn man so will - zweiten Negation aufgeworfen werden, nämlich in welcher Weise dieses Vermögen sich auf phänomenaler d.h. leiblicher Ebene manifestieren kann, ohne dass aber der Mensch erneut bloße Erscheinung, Produkt der Vergangenheit wird? Anders formuliert, geht es darum, einen Platzhalter oder eine Offenheit in der Struktur zu finden, durch welche hindurch sich ein kreatives und auf gelebtes Dasein beruhendes Vermögen ausdrückt. Benjamin schreibt dazu, dass es sich dabei um ein Eingeschränktes, aber nicht Gestaltetes handeln muss, sowohl phänomenal wirksam wie auch nicht durch diese relationale Ebene vollends bestimmbar. „Jene eingeschränkte Realität, welche durch die Fundierung einer geistigen Natur in einem Körper konstituiert wird, heißt die Person".43

\section{Die Person zwischen Leib und Körper}

Der Begriff der Person hat nun zwei Implikationen, von denen nur eine für den Zusammenhang der Wahrnehmung unmittelbar von Bedeutung ist. Die erste Ausdeutung, die an dieser Stelle nicht weiter erläutert wird, ist jene der Bindung an das Gesetz, nämlich als Rechtsperson. In einen universellen sprachlichen Zusammenhang, nämlich jener der gelebten Gemeinschaft in der Welt der Erscheinungen, ist die Person durch einen Beziehung auf das Recht und somit als ethischer (und später bei Benjamin noch stärker politischer) Akteur zu kennzeichnen. Wie von Christoph Menke gezeigt, kann sich eine Betrachtung, die vom ethisch-rechtlichen Problem der Person oder des Menschen ausgeht, auf „Außerrechtliches nur in der Form des Rechts beziehen" 44 , wodurch ein weiteres Vorantreiben der Untersuchung der Wahrnehmung verunmöglicht würde, da alles Nicht-Rechtliche Setzung des Rechts ist. In dem bisherigen Zusammenhang soll die Person gewissermaßen von der anderen Seite aus befragt werden, nämlich wie aus einem singulären oder anonymen Leben, welches den Menschen in seiner Körperlichkeit auszeichnet, ein Ausdruck dessen wird, was der Mensch jeweils sein kann. Die zweite Bedeutung der Person ist daher jene der Maske oder - wie Benjamin später schreibt - „das, wohindurch es hallt" 45

Aus Sicht des Rechts ist die Person, wie erwähnt wurde, Platzhalter für das jeweilige Individuum; seitens der anonymen oder „präpersonellen“ Wahrnehmung aber ist die Person die Möglichkeit des Ausdrucks. Im Leib erscheint der Mensch

43 GSVI, S. 80.

44 Menke, Christoph: Recht und Gewalt, 2. Auflage, August Verlag, Berlin 2012, S. 38.

45 GSII-1, S. 347. 
als wirkliche Person, als konkretes Individuum mit einem konkreten Gesicht. Es ist der Status der Person, der für ein anonymes Leben eine Subjektwerdung erzwingt, der verlangt, etwas für alle anderen Erscheinungen zu sein. Ab dem Moment, indem ein Mensch als Person anerkannt ist, gibt es nur noch den Druck, sich zu verhalten. Daher ist auch das „,höchste moralische Interesse des Subjekts“, wenngleich auch unmöglicher Wunsch, jenes, „sich selbst anonym zu bleiben“ 46

Denn, wie die Beschreibung des Körpers bereits andeutete, kann keine Anerkennung des Körpers im Leiblichen erreicht werden; es könnte auch gesagt werden, dass jede Gestalt des Menschen, wo an ihr festgehalten wird, verfehlte Anerkennung ist. Der Begriff der Person aber bewahrt diesen Status auf, weil er eine Dialektik der Maske einführt, die sich einer mythischen Festschreibung dessen, was als Mensch erscheint, widersetzt.

Die persona (ab hier wird Person und Maske synonym verwendet) ist eine bestimmte Gruppierung von Attributen, die festlegen, was den jeweils konkreten Mensch ausmachen beziehungsweise stellt die Person die jeweiligen Kriterien bereit, um einen Menschen innerhalb eines bestimmten historischen Wahrnehmungsfeldes zu erkennen. Was ein Mensch ist, ist also historisch bedingt. Gleichzeitig - das zeigte auch das Zitat zur Anonymität des moralischen Subjekts an kann jede Erscheinung des Menschen immer mit einem Verweis auf ein So-Nicht, ein Verweis auf eine verhüllte oder versteckte Wahrheit des Individuums vor dem Hintergrund seiner maskenhaften Erscheinung abgetan werden. ${ }^{47}$ Dieser Verweis oder dieses Interesse ist, wie bereits erwähnt, unerfüllbar. Wie Slavoj Žižek festhielt, ist dasjenige, das sich hinter der scheinbaren Maske verbirgt, nicht einfach die Welt, wie sie wirklich ist. Die Vermutung bezüglich des „wahren Gesichts“ ist vielmehr ein Effekt der Maske selbst, eine Setzung, die ausgehend von dem Erscheinen und seiner Negation geschieht, jedoch völlig strikt an die Maske gebunden bleibt - das Gesicht der Maske. ${ }^{48}$ Genau diese Setzung eines imaginären oder projizierten Andersseins, welche nur als ein Außerhalb des Gegebenen gesetzt wird, ist aber folglich Teil des Problems, weil als bestimmte Negation des Zusammenhangs Teil von selbigem. Biologische oder anderweitige, scheinbar natürliche Festsetzungen des Selbstverständlichen am Menschen, wie sie ausgehend von der bürgerlichen Sprachauffassung suggeriert werden, sind dieser Logik folgend immer ein ideologisches Komplement zur gesellschaftlichen und geschichtlichen

46 GSVI, S. 59.

47 Benjamin erkannte diese Logik bei Baudelaire, dessen „exzentrische Eigenart [...] eine Maske [war], unter der er, man darf sagen aus Scham, die überindividuelle Notwendigkeit seiner Lebensform [...] zu verbergen suchte." GSI-2, S. 690.

48 Vgl. Žižek, Slavoj: The Sublime Object of Ideology, Verso, London/New York 2008, S. 25. 
Situation. Mit der Trias zwischen Leib - Körper - Maske ist es mit Benjamin möglich, diese Sackgasse zu verlassene, indem der Körper, der auf einem anonymen und ungeformten Wahrnehmungsfeld operiert, nur aufgrund seines mimetischen Ausdrucksvermögen durch die Person in der Sprache leiblich-sinnlich zugänglich wird. Dieses Vermögen drückt sich in der Person dadurch aus, dass diese sowohl leer ist, nämlich Platzhalter für einen konkreten Fall bleiben muss, und auf der anderen Seite geschichtlich immer konkret ist, somit niemals bloße Leere zum Ausdruck bringt. Die geschichtliche Subjektivität, wie sie „unterleiblich“ (Benjamin) operiert, findet sich in der ethischen Unterbrechung zwischen geschichtlich konkretem Ausdruck und dessen Begrenzung durch den Akt des Benennens, welcher selbst ein Ausdrucksloses bleibt.

Benjamin führt im Laufe seiner Arbeit mehrere Bezeichnungen ein, um die Veränderung der Wahrnehmung ausgehend von einer bestimmten Situation zu charakterisieren. Die logische Denkfigur entspricht dabei jedoch folgenden Bewegungen.

Der Leib muss in seiner Begrenzung, also in seiner grundsätzlichen, strukturellen Verfasstheit ausgewiesen werden, bspw. die jeweils spezifische Zeitlichkeit, wie Benjamin dies für das Barock oder das 19. Jahrhundert unternommen hat. Eine Modifikation des Leibes darf nicht selbst durch ein positives Element erklärt werden, da dieses sonst wiederum in den gegeben Leib eingeht, wie wir im Fall der bestimmten Negation sahen. Oder aber dieses Element wird als überleibliches Prinzip verstanden, wodurch der Leib zum Mythos verallgemeinert wird. In beiden Fällen aber mystifiziert sich der Leib einmal aus einer vollkommen immanenten Gesetzmäßigkeit, einmal als transzendentes Gesetz und wird zu einem Mythos gemacht. Gegenüber der strukturellen Ausdeutung des Leibes gilt es, die jeweilige Einzigartigkeit durch die sprachliche Form der Struktur zu reflektiert: Reflexion heißt hier aber Rückbezug des Denkens auf die Ausgangsbedingungen, was bereits Distanznahme, Modifikation und Deformation miteinschließt. Mehr als das aber heißt dieses Vorgehen, dass eine bestimmte Epoche als begrenzte Leiblichkeit verendlicht wird. Dabei setzt sich das Denken selbst als Subjekt einem Objekt, d.h. einem begrenzten Gegenstand gegenüber nicht mehr unterschiedslose Einheit von Denken und Sein im Mythos, sondern Spaltung, Loslösung vom geschichtlichen Geschehen, indem letzteres wie Benjamin sagt - zum Gehalt wird. ${ }^{49}$ Das so gesetzte Subjekt, die jeweilige Gegenwart, die ein Vergangenes zu seinem Objekt hat, muss sich selbst ins Vergangene reflektieren. Dadurch entsteht nicht eine objektive Perspektive auf die Gegenwart, sondern

49 GS1-I, S. 227. 
eine Gegenwart, die als Abschluss oder wie Benjamin schreibt als „Untergang“ lesbar wird. Dieses Vorgehen hat also nicht zum Ziel, die Vergangenheit in der Gegenwart oder die Gegenwart in der Vergangenheit aufzulösen beides wären mystifizierende Operation. Wie Stéphane Mosès präzise formuliert hat, ist es die „Kollision von Gegenwart und Vergangenheit“, die „bei Benjamin nach dem Modell der Metapher [funktioniert], bei der das Zusammentreffen von zwei Signifikanten, die zu unterschiedlichen Bedeutungsfeldern gehören, einen dritten ganz neuen Signifikanten entstehen lässt“. ${ }^{0}$

\section{Der Mensch als Spannungsverhältnis zwischen Leib und Körper}

Wie wir sehen konnten, ist der Mensch nur über die Position, die er durchläuft und die er benennend vermittelt in seinen Bestimmungen einzuholen, nicht aber als voraussetzungslose Entität erkennbar. Was den Menschen ausmacht, kann nicht aus einem Diskurs stammen, der sich ausnimmt, menschlich zu sein, wie etwa eine eine bloß naturwissenschaftliche Zuschreibung. In einem solchen Fall handelt es sich nämlich um äußerliche Zuschreibungen auf den Menschen hin, in denen das Aussagen nicht auf das Ausgesagte bezogen wird und nur letzte als allgemein gültige Erkenntnis anerkannt wird. Auf der Unterscheidung zwischen Akt des Aussagens bzw. Benennens und der Aussage oder dem Gemeinten beruhend lässt sich somit im Leib die Gesamtheit des Ausgesagten einer Epoche beschreiben, welches genau in jenem Fall mythisch oder gewalttätig ist, wo das Aussagen als Akt nicht mit in die Betrachtung einfließt. Benjamins Unterscheidung zwischen dem Gemeinten und der Art des Meinens scheint in einer analogen Beziehung zu diesen zwei Ebenen zu stehen. Der Leib wäre damit die Allgemeinheit des Gemeinten einer Epoche, die Art des Meinens entspräche demgegenüber dem körperlichmimetischen Akt des Benennens. In der Spannung zwischen Benennen und damit Urteilen und Unterscheiden, aber auch Vervielfältigen und Differenzieren der phänomenalen Welt auf der einen Seite, die immer wieder unvollständige Wiederholung des Benannten im Akt des Nennens und Neubenennens auf der anderen Seite, ist vor diesem Hintergrund die Bewegung des Denkens oder des Lebens, welches sich in der immer unvollständigen, ständig aber nach Gesetzmäßigkeiten verhaltenden Logik einer geschichtlichen Epoche oder „Monade“ formiert, konfiguriert und wandelt. Der Mensch ist in diesem Zusammenhang als die offene aber

50 Mosès: „Eingedenken und Jetztzeit - Geschichtliches Bewußtsein im Spätwerk Walter Benjamins“, in Havelkamp, A. (Hrsg.), Memoria. Vergessen und Erinnern, Wilhelm Fink Verlag, München 1993, S. 395 . 
unteilbare Einheit der Idee einer Epoche zu denken und nur in und durch eine solche zu bestimmen.

Menschenähnlichkeit heißt folglich, dass der Mensch sich unverständlich geworden ist: wenn das, was der einzelne Mensch ist, wie er erscheint, nicht mehr selbstverständlich ist, dann ist dies aber keinesfalls eine bloß negative Funktion. Vielmehr deutet eine solche „Entselbstverständlichung" auf einen Bruch mit dem/ einem traditionellen Menschenbild hin. Eine vorherrschende Vorstellung vom Menschen hört auf, Gemeinplatz zu sein und ein Ersatz ist noch nicht gefunden. In diesem Moment, wo die Menschenähnlichkeit oder die Ähnlichkeit des Menschen mit sich aufhört, reibungslos zu operieren, eröffnen sich Möglichkeitsräume der Selbsttransformation. Doch ist ein solcher Moment eine offene Situation, die gegen die Normalisierung durch die Tradition oder die Nivellierung des Bruches verteidigt werden muss. Um den einzelnen Menschen in seiner Singularität gerecht zu werden, muss dieser Moment des Bruchs „Augenblick der Gefahr" ${ }^{\text {51 }}$ wahrgenommen werden und gegen eine Einverleibung in eine homogene Geschichtlichkeit verteidigt werden. Wenn Benjamin daher später schreibt, dass der Mensch auch bereit sei, die Kultur zu überleben, wenn es sein muss, ${ }^{52}$ so zielt er auf eben jene demystifizierende Operation ab, die das gelebte Leben dem Mythos entreißen kann und dem Menschen die reelle Möglichkeit zur ethisch verantwortlichen Selbsttransformation eröffnet.

Philipp Nolz studierte Philosophie sowie Wirtschafts- und Sozialwissenschaften in Wien und Istanbul. Derzeit ist er als Doktorand an der Bergischen Universität Wuppertal und an der Université Paris 8 Vincennes - St. Denis tätig, wo er zum Erfahrungsbegriff in der Geschichtsphilosophie Walter Benjamins schreibt.

51 GSI-2, S. 695.

52 GSII-1, S. 219. 